\title{
New Clues to Breast Cancer Heterogeneity
}

\author{
Hans Kreipe \\ Institute of Pathology, Medizinische Hochschule, Hanover, Germany
}

Breast Cancer is a heterogeneous disease. To delineate heterogeneity and to exploit it as a principle for prognostic and therapeutic stratification still represents a major challenge in breast cancer research. Traditionally, in most cancers histological typing and grading yields the basis for definition of subgroups. In breast cancer this stage of development has long been passed and novel principles are emerging. Gene expression profiling has enabled the definition of so-called 'intrinsic subtypes' of breast cancer. A decade ago the seminal study of Perou and co-workers [1] has paved the way to molecular classification. Although only a low number of cases were studied, the classification into luminal A and B type, basal type and HER2 type provided a useful frame for clinical decision-making. In fact, this classification was not completely new but reflected the expression of markers which are routinely assessed anyway in breast cancer by immunohistochemistry. When molecularly defined intrinsic types are translated into categories to be assessed by immunohistochemistry two areas of incongruity can be identified. First, there is no validated tool to discriminate between luminal A and B type, although Ki-67 has been suggested by the St. Gallen consensus 2011 [2]. The intrinsic types and their immunohistochemical identification are discussed by Strehl and co-workers in this issue [3].

Second, the so called basal type of breast cancer has no consented counterpart in the realm of immunohistochemistry. What turned out to be a potential substitute under diagnostic and pragmatic aspects is the subgroup of triple negative breast cancer. Both categories share a considerable overlap as pointed out by Zeinab and Sinn in this issue [4]. Although the immunohistochemically defined triple negative type of breast cancer has a more homogenous definition than the basal type, there are still some uncertainties. These refer to the ill-de- fined borders between estrogen receptor positivity and negativity $(0,1$, or $10 \%)$ and the necessary absence of progesterone receptor expression. In addition, the triple negative type of breast cancer encompasses cancer types with heterogeneous histology and biology such as medullary type, myoepithelial cancer and metaplastic cancer. Therefore, the triple negative attribute can clinically be applied only in conjunction with the histological subtype [4].

Unlike microbiologists with antibiotics, pathologists are not able to forecast the sensitivity of a certain cancer to chemotherapy regimens. There is some correlation to histological type and marker expression, but in individual cases the efficacy of chemotherapy cannot be predicted. Neoadjuvant chemotherapy has opened a new window of information on chemosensitivity of cancers in vivo. Denkert and co-workers have conducted a number of studies on histopathological and immunohistochemical markers in neo-adjuvant therapy trials. By comparing core biopsy findings with level of regression in resection specimens they identified markers which are able to predict chemosensitivity with high probability. Their findings and experiences are summarized in this issue [5].

With regard to breast cancer heterogeneity, novel upcoming markers will lead to new categories of breast cancer, but there seems to be no revolution ahead introducing a completely new system. Rather basal categories defined by histology and steroid hormone receptor expression will persist in the future, and to this framework subgroups will be added, defined by methods which can broadly be applied.

\section{Disclosure Statement}

The author declares that he has no conflict of interests.

\section{References}

1 Perou CM, Sørlie T, Eisen MB, van de Rijn M, Jeffrey SS, Rees CA, Pollack JR, Ross DT, Johnsen H, Akslen LA, Fluge O, Pergamenschikov A Williams C, Zhu SX, Lønning PE, Børresen-Dale AL, Brown PO, Botstein D: Molecular portraits of human breast tumours. Nature 2000;406:747-752.

2 Goldhirsch A, Wood WC, Coates AS, Gelber RD, Thürlimann B, Senn HJ; Panel members: Strategies for subtypes--dealing with the diversity of breast cancer: highlights of the St Gallen International Expert Consensus on the Primary Therapy of Early Breast Cancer 2011. Ann Oncol 2011;22:1736-1747.

3 Strehl JD, Wachter DL, Fasching PA, Beckmann MW, Hartmann A: Invasive breast cancer: recognition of molecular subtypes. Breast Care 2011;6: 258-264.
4 Elsawaf Z, Sinn HP: Triple negative breast cancer: clinical and histological correlations. Breast Care 2011;6:273-278.

5 Denkert C, Sinn, BV, Issa Y, Müller BM, Maisch A, Untch M, von Minckwitz G Loibl S: Prediction of response to neoadjuvant chemotherapy new biomarker approaches and concepts. Breast Care2011;6:265-272.

\section{KARGER}

Fax +497614520714

Information@Karger.de

www.karger.com (c) 2011 S. Karger GmbH, Freiburg

$1661-3791 / 11 / 0064-0256 \$ 38.00 / 0$

Accessible online at:

www.karger.com/brc
Prof. Dr. med. Hans Kreipe

Institut für Pathologie, Medizinische Hochschule,

Carl Neuberg Str. 1

30625 Hannover, Germany

Tel. +49 511 532-4500 Fax -5799

Kreipe.Hans@MH-Hannover.de 